\title{
The Nature of the X-Ray Point Source in the Bar of NGC 6822
}

\author{
Paul B. Eskridge \\ and \\ Raymond E. White III \\ Department of Physics and Astronomy, \\ University of Alabama, Tuscaloosa, AL 35487
}

\begin{abstract}
We have analysed archival ROSAT PSPC and Einstein HRI data for the $\mathrm{x}$-ray point source in the bar of NGC 6822. The source decreased in x-ray flux by at least half an order of magnitude in the 13 years between the HRI and PSPC observations. It has a PSPC flux of $f_{X} \approx 8 \times 10^{-14} \mathrm{erg} \mathrm{cm}^{-2} \mathrm{~s}^{-1}$, giving $L_{X} \approx 2.4 \times 10^{36} \mathrm{erg} \mathrm{s}^{-1}$ for a distance of $500 \mathrm{kpc}$. The source appears to be related to the optical emission-line object Ho 12. It is unclear if Ho 12 is an H II region or a supernova remnant, although the latter interpretation is better supported by the available optical data. The x-rays are unlikely to be direct emission from a supernova remnant, due to the observed strong x-ray variability. The x-ray spectrum of the source is very soft. Acceptable fits are found for either a Raymond-Smith thermal model with $\mathrm{kT} \approx 0.56 \mathrm{keV}$, or a blackbody model with $\mathrm{kT} \approx 0.06 \mathrm{keV}$. The latter model would place the source amongst the class of Super-Soft x-ray sources first found in the Magellanic Clouds. If the thermal model is correct, the source appears similar to Galactic and Magellanic Cloud stellar-mass black hole binary candidates. We have identified a list of potential optical counterparts from published photometry. However, for either model discussed above, we expect the apparent magnitude of the optical counterpart to be in the range $21.5 \lesssim m_{V} \lesssim 24.0$. Thus the optical counterpart may be below the limit of published photometry.
\end{abstract}

\footnotetext{
${ }^{1}$ paul@hera.astr.ua.edu

${ }^{2}$ white@merkin.astr.ua.edu
} 


\section{Introduction}

NGC 6822 is the nearest dwarf irregular (dI) galaxy beyond the Galactic halo, and has the distinction of being the first object clearly identified as an external stellar system (Perrine 1922; Hubble 1925). It has a Cepheid-based distance modulus of $(m-M)_{\circ}=23.5$ (McAlary et al. 1983; Gallart, Aparicio \& Vílchez 1996a), corresponding to a distance of $\sim 500 \mathrm{kpc}$. Dwarf galaxies in the Local Group have much less rich x-ray source populations than do the more luminous galaxies, and they have therefore been relatively neglected. This is the third in a series of papers that seek to redress this situation. Previous papers in the series studied IC 1613 (Eskridge 1995) and M32 (Eskridge, White \& Davis 1996).

There have been a large number of studies of the stellar populations of NGC 6822, including global studies of the luminous stars (e.g. Hodge et al. 1991 and references therein), and much deeper CCD studies of selected areas (e.g. Wilson 1992a; Gallart et al. 1996a). All these studies show the young population quite clearly: there is a strong blue main-sequence (MS), as well as yellow and red supergiants. The existence of a substantial intermediate age population (from the presence of an extended asymptotic giant branch AGB) is most obvious from the VI photometry of Gallart et al. (1996a). While there is no unambiguous evidence for a true Pop II in the field of NGC 6822, the study of Gallart et al. (1996b) strongly supports the existence of such a population. There are $\sim 20$ OB associations identified in NGC 6822 (Hodge 1977; Wilson 1992a). Wilson (1992a) presents color-magnitude $(\mathrm{CM})$ diagrams for 13 of these, down to $V \approx 22$. These CM diagrams are only deep enough to show the OB main sequence stars and the supergiants. Wilson finds a slope of the upper MS comparable to that found in IC 1613 (Freedman 1988), and other Local Group galaxies (e.g. Freedman 1985), although Massey et al. (1995) argue that NGC 6822 is deficient in both the most massive stars and the most populous OB associations compared to the Galaxy and the Magellanic Clouds (MCs).

The optical structure of NGC 6822 has been studied by Hodge (1977) and Hodge et al. (1991). The system is dominated by a strong bar oriented roughly north-south. The brightest H II regions are to the north of the bar. Markert \& Donahue (1985) provide an excellent image (their Fig. 1) showing the overall optical structure of the system. The absolute magnitude of NGC 6822 is $M_{B} \approx-15.9$, just over a magnitude fainter than the SMC. Hodge et al. (1991) find that the density of resolved stars (from Schmidt plates), as well as the light from the unresolved stars can be fit with an exponential with a scale length of $\sim 120^{\prime \prime}$. They also find a limiting radius of $\sim 40^{\prime}$. deVaucouleurs et al. (1991, hereafter RC3) quote a major axis diameter of $D_{25}=15.5$.

The H I structure and content of NGC 6822 has been studied by Skillman (1987) and Gottesman \& Weliachew (1977). NGC 6822 has $M_{H I} / L_{B} \approx 0.6$ (in Solar units), and its 
$\mathrm{H}$ I emission is far more extended than its optical emission. These are typical properties for a dI galaxy. NGC 6822 is unusual in that the optical and H I major axes are misaligned by almost $90^{\circ}$ (see Fig. 11 in Hodge et al. 1991). CO emission has been detected at a number of locations in NGC 6822 (e.g.Wilson 1992b; Ohta et al. 1993; Wilson 1994; Israel, Tacconi \& Baas 1995). In general, the CO peaks are associated with either the brightest H II regions, or with peaks in the H I surface density. NGC 6822 has a rich population of H II regions, indicating substantial recent and on-going star formation. The most complete study of the H II regions of NGC 6822 is that of Hodge and collaborators (Collier, Hodge \& Kennicutt 1995, and references therein). NGC 6822 is near enough that it is resolved in the IRAS far-infrared (FIR) data (Gallagher et al. 1991). There is a general correspondance between the FIR emission and both the $\mathrm{H}$ II regions and peaks in the $\mathrm{H}$ I distribution, with some evidence for diffuse FIR emission as well.

The two main techniques for estimating heavy element abundances in Local Group objects are spectrophotometry of nebular emission-line objects, and isochrone fitting onto color-magnitude diagrams (CMDs) of resolved stellar populations. For NGC 6822, studies of H II regions (Skillman, Terlevich \& Melnick 1989) and planetary nebulae (Richer \& McCall 1995) indicate similar oxygen abundances. The H II region result is $12+\log (O / H)=8.20$, and the planetary nebulae result is $12+\log (O / H)=8.1 \pm 0.1$. This corresponds to $0.15-0.20 Z_{\odot}$, adopting $12+\log (O / H)_{\odot}=8.93$ (Grevesse \& Anders 1989). The CMD study of Gallart et al. (1996b) is the best available for isochrone fitting. They conclude that their data support $[\mathrm{Fe} / \mathrm{H}] \approx-1.0$ for the most metal-rich population. Given the systematic ambiguities of relating gas-phase oxygen abundances to mean metallicities from isochrone fitting to resolved stellar populations, these results are in reasonable agreement.

The x-ray populations of the Galaxy and the MCs have been extensively studied with all available x-ray telescopes over the last several decades (e.g. Snowden 1996; Verbunt 1993; Bradt, Ohashi \& Pounds 1992; Wang \& Wu 1992; Wang et al. 1991). There have also been studies of the x-ray source populations in M31 (e.g. Supper et al. 1997; Primini, Forman \& Jones 1993; Fabbiano, Trinchieri \& van Speybroeck 1987) and M33 (e.g. Long et al. 1996; Trinchieri, Fabbiano, \& Peres 1988). As noted above, there has been less work on the more optically faint galaxies in the Local Group. The x-ray emission from M32 is dominated by an unresolved source that may be emission from low-mass x-ray binaries (LMXRBs) or a $\mu \mathrm{AGN}$ (Eskridge et al. 1996). The x-ray emission in the direction of the Local Group dI IC 1613 is dominated by a background galaxy cluster (Eskridge 1995).

The organization of this paper is as follows: In $\S 2$ we review the existing x-ray data for NGC 6822. These data reveal a single bright point source, and no evidence for diffuse emission. In $\S 3$ we examine the optical information on the environment of the x-ray point 
source. We propose various interpretations for the nature of the source in $\S 4$. In $\S 5$ we discuss these possibilities, and outline the observations required to distinguish between them. Finally, $\S 6$ presents a summary of our results.

\section{X-Ray Observations}

NGC 6822 was observed with the Einstein Observatory HRI (Giacconi et al. 1979) for 34.4 kiloseconds on 31 Oct. 1979 (Markert \& Donahue 1985). This observation detected a bright point source in the direction of the bar, and another source that appears to be in the background (see Fig. 1 in Markert \& Donahue 1985; see also Fig. 7 of Fabbiano, Kim \& Trinchieri 1992). Markert \& Donahue (1985) point out that the source in the bar falls within the emission line object Ho 12 (Hodge 1977), and suggest that the x-ray source is related to this object. The count-rate of the source from the Einstein HRI exposure is $0.0043 \mathrm{~s}^{-1}$. For a foreground column of $N_{H}=9 \times 10^{20} \mathrm{~cm}^{-2}$ (Stark et al. 1992), and assuming a $5 \mathrm{keV}$ bremmstrahlung spectrum (we shall return to this point shortly), this corresponds to a flux of $8.56 \times 10^{-13} \mathrm{erg}^{-2} \mathrm{~cm}^{-1}$ in the $0.2-4.0 \mathrm{keV}$ band (or a luminosity of $\left.L_{X} \approx 2.6 \times 10^{37} \mathrm{erg} \mathrm{s}^{-1}\right)$. This is a perfectly reasonable luminosity for a LMXRB (e.g. White, Nagase \& Parmar 1995).

The source was reobserved with the ROSAT PSPC in 1992 (sequence \#RP600148). Although the right ascensions from the HRI and PSPC observations agree to $\sim 1^{\prime \prime} .5$, the declinations differ by $\sim 12^{\prime \prime}$, with the PSPC position north of the HRI position. This is larger than the nominal positional accuracy of either instrument, and is thus a cause for some concern. We found that the second HRI source was also recovered in the PSPC observation, and showed the same offset in declination. Thus there is a systematic offset in the aspect solutions for the two observations. Neither source has any obvious optical or radio counterpart, however there are seven additional sources detected in the PSPC observation. None of these additional sources are coincident with any cataloged radio source, or with any optical source down to the sky survey limit. However, we find that two of these sources (1RXP J194427-1443.0 and 1RXP J194505-1436.4) are 12" north of moderately bright stars (once again, there are no cataloged radio counterparts). From this conclude that the systematic error is in the PSPC astrometry for the field, perhaps due to the same problem discussed by Harris, Perley \& Carilli (1994).

The PSPC exposure was quite short; the dead-time corrected exposure is $6364 \mathrm{~s}$, resulting in $\sim 75$ total source counts. This corresponds to a PSPC count-rate of $0.0012 \mathrm{~s}^{-1}$. We extracted the source spectrum from a $2^{\prime}$ radius circle, centered on the source. We used a

background spectrum drawn from an annular region, also centered on the source, extending 
from $2^{\prime}$ to 3'75. We used XSPEC v9.0 (Arnaud 1996) to assess a variety of spectral models. However, due to the small number of source counts, the spectral parameters are rather poorly determined. Statistically acceptable fits exist for both Raymond-Smith thermal (RS) and blackbody (BB) models (see Table 1). The RS model yields acceptable fits for two distinct temperatures, with $\mathrm{kT} \approx 0.04 \mathrm{keV}$ for the soft solution, and $\mathrm{kT} \approx 0.56 \mathrm{keV}$ for the hard solution. The BB model gives an acceptable fit for the soft solution only (with $\mathrm{kT} \approx 0.06 \mathrm{keV}$ ). As noted above, the observed Galactic column in this direction is $N_{H}=9 \times 10^{20} \mathrm{~cm}^{-2}$. The observed intrinsic column associated with this region of NGC 6822 is $N_{H} \approx 2 \times 10^{21} \mathrm{~cm}^{-2}$ (see Hodge et al. 1991). Thus we can constrain good fits to have $N_{H}$ in the range $\sim 1-3 \times 10^{21} \mathrm{~cm}^{-2}$. This argues against the soft RS fit unless the source is strongly self-absorbed. Both the hard RS fit and the BB fit have $N_{H}$ in the acceptable range. In Figure 1, we show the observed spectrum, along with the fits for the $0.56 \mathrm{keV}$ $\mathrm{RS}$ model and $0.06 \mathrm{keV} \mathrm{BB}$ model. We show the $\chi^{2}$ confidence contours of $N_{H}$ and $\mathrm{kT}$ for these models in Figure 2. The contours are at 1 $\sigma, 90 \%$, and $99 \%$ levels. In particular, we note that a hot, highly absorbed model is strongly ruled out by the data.

The spectral models discussed above yield a flux of only $\sim 8 \times 10^{-14} \mathrm{erg} \mathrm{cm}^{-2} \mathrm{~s}^{-1}$ in the $0.2-2.0 \mathrm{keV}$ band (this value changes by $\lesssim 10 \%$ when computed for the Einstein band). This is an order of magnitude lower than the published Einstein flux. However the Einstein flux was computed for an assumed spectral model that is quite different from (and much harder than) those we find for the PSPC data. We tested the importance of this difference by using the spectral models that fit the PSPC data to predict the Einstein HRI count-rate a posteriori. The results of this experiment are given in Table 1; for all good PSPC spectral models, the predicted Einstein HRI count-rate is still roughly half a dex lower than the observed Einstein HRI count-rate. Thus the source is x-ray variable by at least half a dex on $\sim$ decade timescales. We searched for evidence of short-term variability in the PSPC data by inspecting the count rates for the individual observation intervals. Any variability in the data can be easily explained by fluctuations in the background. This is not a terribly strong constraint, given the small number of source counts.

\section{Source Environment}

\subsection{Association with Ho 12}

As the source is superposed on the bar of NGC 6822, one might expect it to be an LMXRB. However, as LMXRBs tend to have temperatures of $3-5 \mathrm{keV}$ (e.g. White et al. 1995), the PSPC data argue strongly against this interpretation. As noted by Markert \& Donahue (1985), the source is projected on the emission-line object Ho $12: \Delta \alpha=0 s \cdot 2$, 
$\Delta \delta=4^{\prime \prime}$, where we take the x-ray position from Fabbiano et al. (1992), and the Ho 12 region position from Hodge, Kennicutt \& Lee (1988). In Table 2, we give these positions precessed to J2000 coordinates. As noted in $\S 2$, above, this places the x-ray source within the boundaries of Ho 12. Figure 3 is an $I$-band image centered on the x-ray source that was kindly provided by C. Gallart. The region shown is $54^{\prime \prime}$ on a side. The contours are $\mathrm{H} \alpha$ emission from Collier \& Hodge (1994). The circle centered on the x-ray position is $10^{\prime \prime}$ in radius, and contains the stellar objects listed in Table 3. These stars are identified from the photometry of Gallart et al. (1996a). Positions for the stars in Table 3 were computed from the astrometry of Wilson (1995) for nearby stars common to the Wilson (1992) and Gallart et al. (1996a) studies. Our positions are on Wilson's (1995) astrometric system to $\sim 1^{\prime \prime}$. We also give magnitudes and colors for the stars from the photometry of Gallart et al. (1996a), and the offsets between the stellar positions and that of the x-ray source. There are 17 stars listed in Table 3 . Not all of these stars have measured $I$-band magnitudes, and so not all are visible on Figure 3. A number are visible, but are too severely blended for accurate photometry.

\subsection{The Nature of Ho 12}

Ho 12 was identified as an emission-line object by Hodge $(1969 ; 1977)$, and is included in the study of the properties of the H II regions of NGC 6822 by him and his collaborators (Collier et al. 1995). It is, however, unclear if this object is an H II region or a supernova remnant (SNR). Smith (1975) obtained an IDS spectrum of Ho 12 with a wavelength coverage of $\sim 2400 \AA$, from $\mathrm{H} \beta$ to the $[\mathrm{S}$ II] $\lambda \lambda 6717,6731 \AA$ doublet (he uses the Hodge 1969 nomenclature, and thus refers to the object as \#10. This is the same object as Ho 12 , following Hodge 1977). He did not publish his spectrum, however he states that the [S II] doublet ratio implies a high electron density, that the $[\mathrm{S} \mathrm{II}]$ to $\mathrm{H} \alpha$ ratio is quite high $(\sim 0.5)$ and that $[\mathrm{O} \mathrm{I}] \lambda 6300 \AA$ is observed. All of these argue against Ho 12 being a typical H II region.

D’Odorico, Dopita \& Benvenuti (1980) obtained narrow-band photographic images of Ho 12 in $\mathrm{H} \alpha$ and [S II] with the 48" Schmidt telescope at Palomar Observatory. Considering both their imaging and Smith's spectrophotometry, they argue that Ho 12 is actually an SNR. They state that subsequent AAT spectroscopy confirm this designation, but these data have never been published. The case for Ho 12 being an SNR is reasonable, but it would be very useful to obtain new spectrophotometry.

Wilson (1992a) identifies an OB association (her OB11) containing Ho 12 (Hodge's (1977) OB10 is a subset of her OB11). Based on her CM diagram (shown in her Fig. 6), 
she estimates an age of $\sim 8 \times 10^{6}$ yr for OB11. There are stars with $(B-V) \approx 0$ as bright as $V \approx 18$ in her CMD. These stars have absolute magnitudes of $M_{V} \approx-5.5$, roughly commensurate with O5 stars. There are thus clearly stars hot and luminous enough to support an H II region as bright as Ho 12. There is also a rich population of red supergiants (reaching $V \approx 18,(B-V) \approx 2$ ), demonstrating that the star-forming event began sufficiently long ago for the most massive stars to have already gone supernova. Thus the stellar population in the vicinity does not offer any firm guidance for deciding if Ho 12 is an H II region or an SNR. We will therefore consider both interpretations in our analysis below.

\section{Possible Interpretations}

The source is far too cool to be a typical high-mass x-ray binary (HMXRB), as such sources typically have $\mathrm{kT} \approx 10 \mathrm{keV}$ (e.g. White et al. 1995). SNR can have the sort of x-ray spectral properties observed (e.g. Seward, Schmidt \& Slane 1995). The evidence that Ho 12 is an SNR makes this a possibility well worth considering. There is no radio continuum emission from the source down to limits of $25 \mathrm{mJy}$ at $21 \mathrm{~cm}$ (Gottesman \& Weliachew 1977), $42 \mathrm{mJy}$ at $6 \mathrm{~cm}$ (Griffith et al. 1994), and $2.7 \mathrm{mJy}$ at $2.8 \mathrm{~cm}$ (Klein, Gräve \& Wielebinski 1983). This is probably not a strong constraint: Mathewson et al. $(1983,1985)$ present radio continuum and x-ray data for a sample of MC SNR. We use their data to define a typical range in $\mathrm{x}$-ray flux to radio power for SNR. This range is $\sim 2 \mathrm{dex}$, thus it has only rough predictive power. Mathewson et al. $(1983,1985)$ give radio data at either 408 or 843 MHz. We correct this to the observed frequencies for NGC 6822 by taking a typical SNR radio spectral index of $\alpha \approx-0.5$. The result of this excercise is that the expected radio power of a SNR with the observed $f_{X}$ is on the order of the current observational limits, if it has properties like those of the SNR in the MCs.

A more compelling argument against the x-rays being from an SNR is the x-ray variability between the Einstein and ROSAT observations. Of course, this assumes that the x-rays observed by both Einstein and ROSAT come from the same object. It is possible that the Einstein data are dominated by emission from a highly variable compact x-ray source that was quiescent during the PSPC observation, and that the PSPC data are entirely due to emission from Ho 12. The $\mathrm{H} \alpha$ emission from Ho 12 is clearly extended (see Figure 3); the main structure has a diameter of $\sim 10^{\prime \prime}$. This is substantially less than the point-spread function of the ROSAT PSPC, thus we would not expect the PSPC emission to be resolved (nor do we observe it to be) even if it were due to emission from Ho 12 . The Einstein HRI emission is unresolved, but a small enough fraction of the HRI counts 
would be due to the extended source that this is not a useful constraint. Although such a multi-source model is unappealing when viewed from the edge of Occam's razor, the available data do not rule it out. One way of testing this two-source picture would be to obtain a deep ROSAT HRI image to search for evidence of extended emission. Keeping this in mind, we turn our attention to possible interpretations for the emission assuming that only one source of x-rays is present.

\subsection{Is the Object a Super-Soft Source?}

We suggest that this source may be a "Super-Soft" source (SSS), such as were originally discovered in the MCs (Long, Helfand \& Grabelsky 1981). X-ray emission from these sources is best fit by optically thick (blackbody) models, with temperatures of $\sim 50 \mathrm{eV}$, and x-ray luminosities up to $\sim 10^{38} \mathrm{erg} \mathrm{s}^{-1}$ in the ROSAT band. The PSPC data for the NGC 6822 source are entirely consistent with these spectral properties. The luminosity of the source is a bit low in the PSPC observation, but the source was $\sim 10$ times brighter in the Einstein HRI observation; the maximum observed luminosity $\left(L_{X} \approx 2.6 \times 10^{37} \mathrm{erg} \mathrm{s}^{-1}\right)$ is in keeping with observations of other SSSs. Also, recent observations of CAL 83 discussed in Alcock et al. (1997) have shown it to exhibit strong x-ray variability. This may be a typical property of SSSs.

The nature of SSSs is still unclear. There are several currently debated models for SSSs, and it is likely that they are not a homogenous class (e.g. Kahabka, Pietsch \& Hasinger 1994). One idea is that SSSs are due to high accretion-rate nuclear burning on white dwarfs (e.g. Kahabka 1995). This model is particularly well developed for the transient SSSs. Another proposed mechanism is emission from the hot inner part of white dwarf accretion disks. It has also been proposed that SSSs are due to accretion onto black holes, or onto neutron stars. Especially in the case of neutron stars, this requires the energy source to be enveloped in an optically thick cocoon of material in order to produce x-rays as soft as those observed. See Crampton et al. (1996) and Kahabka et al. (1994) for further discussion of these models, and references to earlier work.

\subsection{Is the Object a Stellar-mass Black Hole Binary?}

The spectrum can also be fit with a model that is substantially hotter than any appropriate for a SSS. The hotter fit has spectral properties similar to those observed for a number Galactic and MC stellar-mass black hole binary candidates (e.g. Cowley 1992 and 
references therein). Thus, if the hotter fit is correct, the best working hypothesis for the source is that it is a black hole binary system. The x-ray spectral properties of black-hole binary candidates are heterogenous (viz. Cowley 1992; McClintock, Horne \& Remillard 1995). While they typically have fairly soft spectra $(\mathrm{kT} \lesssim 1.5 \mathrm{keV}$; indeed, the SSS CAL 87 is a good black-hole binary candidate, as discussed by Cowley 1992), some sources exhibit substantial x-ray spectral variability, and can have states with spectra characterized by temperatures of up to $\mathrm{kT} \approx 30 \mathrm{keV}$ (e.g. V616 Mon).

\section{Discussion}

\subsection{Optical Identification and Possible Follow-up Observations}

In $§ 3.1$ we presented information from the photometry of Gallart et al. (1996a) on 17 stars within $10^{\prime \prime}$ of the x-ray position. Observations of Galactic and MC SSSs and black-hole binary candidates show them to typically have $-2 \lesssim M_{V} \lesssim+0.5$ and $(B-V) \approx 0$ (e.g. Crampton et al. 1996; Beuermann et al. 1995; Cowley et al. 1993; Cowley 1992 and references therein). For an object at the distance of NGC 6822, the above apparent magnitude range corresponds to $21.5 \lesssim m_{V} \lesssim 24.0$. The faintest objects in Table 3 have $m_{B} \approx 22.5$, thus it is entirely possible that the optical counterpart to the x-ray source is undetected in the existing photometry. Among the stars listed in Table 3, the most likely candidates for the optical counterpart are those with the bluest colors, and within $5^{\prime \prime}$ of the x-ray position: Stars 17801, 17896, 17996, 18260, and 18332. We are seeking to obtain follow-up spectroscopy of these candidates. While this is clearly warrented, deeper photometry from HST is likely to be required to find the optical counterpart. Work on the UV properties of Galactic and MC SSSs and black-hole binary candidates (e.g. Treves et al. 1990; Beuermann et al. 1995) argues that the object will have an unabsorbed near-UV

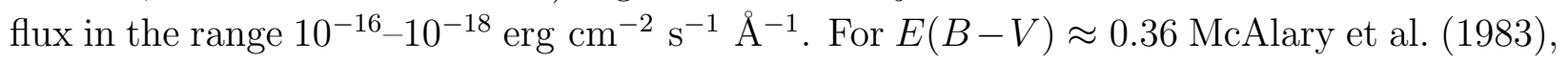
a STIS NUV image of the region centered on the $\mathrm{x}$-ray position should detect the source at the $10 \sigma$-level, even in it's faint state, in an integration time of $\sim 2000$ seconds. The OB 11 turn-off stars in the vicinity have $V \approx 21$. These stars should also be easily detected in such an NUV image, making it possible to tie the astrometry between the optical and UV observations, and unambiguously identify the UV sources in the field. If the source is UV-bright $\left(\sim 10^{-16} \mathrm{erg} \mathrm{cm}^{-2} \mathrm{~s}^{-1} \AA^{-1}\right)$ it is likely to be a black-hole binary. If it is closer to the faint end of the range, this would support the SSS interpretation. We plan on proposing for such observations in order to study the properties of the source in more detail.

It would be interesting to obtain better x-ray data on the NGC 6822 source. As noted in $\S 4$, a deep ROSAT HRI observation could test the possibility that there are two sources 
of x-rays, one being faint extended non-variable emission from Ho 12, and the other being compact highly variable emission from an x-ray binary. ASCA, RXTE, or SAX observations could, in principle, provide much better spectral constraints than the PSPC data. However, the soft spectrum poses something of a problem, especially if the source happens to be in a low-flux state when observed. As an illustrative example, if the source were observed for $20 \mathrm{ksec}$ by $A S C A$, while in its low-flux state, the resulting spectrum would provide no improvement over the $\sim 6 \mathrm{ksec}$ PSPC spectrum. Similar arguments hold for RXTE and $S A X$. It is unlikely that improved x-ray spectral data for the NGC 6822 source will be obtained until $A X A F$ is in orbit.

\subsection{Comparison of NGC 6822 with Other Local Group Galaxies}

It is useful to compare the x-ray properties of NGC 6822 with those of the MCs and M31. The MCs are low-luminosity star-forming systems much like NGC 6822, but are somewhat more luminous, and a great deal closer. Their x-ray properties have therefore been studied in some detail. M31 is the closest giant galaxy, and has also been well studied in the x-ray.

The x-ray emission in NGC 6822 and the MCs is mainly due to a very small number of discrete sources. Thus we compare the source populations of the three systems. In order to do so, we adopt the total blue magnitudes for all three galaxies as given in the RC3 $\left(B_{T}^{0}=0.57,2.28\right.$, and 8.39 for the LMC, the SMC, and NGC 6822 respectively), and distances of $50 \mathrm{kpc}$ for the LMC, and $65 \mathrm{kpc}$ for the SMC (as noted in $\S 1$, the distance to NGC 6822 is $\sim 500 \mathrm{kpc}$ ). Schmidtke et al. (1994) and Cowley et al. (1997) present the current results for the x-ray source populations of the MCs. These studies are based on ROSAT HRI observations, and thus do not provide spectral information. If we scale our observed PSPC count-rate to an expected HRI count-rate for models appropriate for our source, and then correct these count-rates to the MC distances, we find that the source in NGC 6822 would have an HRI count-rate of $\sim 0.040 \mathrm{~s}^{-1}$ were it in the LMC, or $\sim 0.023 \mathrm{~s}^{-1}$ were it in the SMC. Schmidtke et al. (1994) and Cowley et al. (1997) indentify half a dozen sources in each galaxy that are at or above these limits. From the apparent magnitudes and distances given above, the expected numbers are $\sim 13$ in the LMC, and $\sim 5$ in the SMC. Given the small numbers involved, and the crudeness of the comparison, this is a surprisingly good result.

For M31, we adopt a total blue magnitude of $B_{T}^{0}=3.34$ from the RC3, and a distance of $690 \mathrm{kpc}$. Supper et al. (1997) find 200 sources in M31 that are as luminous as the NGC 6822 source. As the optical luminosity of M31 is 200 times that of NGC 6822, this 
comparison suggests that the number of discrete x-ray sources does scale quite well with optical luminosity.

The issue of how galaxy morphology affects x-ray source population is still quite mysterious. The above arguments indicate that low luminosity galaxies are just as efficient at producing stellar x-ray sources as high luminosity galaxies. However, their are other indications that this is not so. ROSAT observation of the Fornax dwarf spheroidal (Gizis, Mould \& Djorgovski 1993) found no evidence of any stellar x-ray sources associated with Fornax. Comparing Fornax's optical luminosity to those of Galactic globular clusters, Gizis et al. (1993) concluded that the extremely low density environment of Fornax is significantly less efficient at producing stellar x-ray sources than are environments such as globular clusters. The problem with all of this is the small number of sources found associated with the small number of galaxies that have been studied. It would be very interesting to determine how the x-ray source populations of diffuse dwarf galaxies (both early- and late-types) differ from those of more luminous systems. We certainly cannot draw any firm conclusions in this paper, but it may be possible to address this issue sensibly by studying the composite x-ray properties of a large sample of dwarf galaxies.

\section{Summary}

A bright x-ray point source in the bar of NGC 6822 was originally discovered in an Einstein HRI image (Markert \& Donahue 1985). A subsequent ROSAT PSPC observation reveals that the source is variable by roughly a dex on the timescale of a decade, and has has a very soft x-ray spectrum. The PSPC observation was quite short ( $\sim 6 \mathrm{ksec})$, resulting in only $\sim 75$ source counts. Thus the spectral properties are not well constrained. We can fit the data with either a $\mathrm{RS}$ model with $\mathrm{kT} \approx 0.56 \mathrm{keV}$, or with a $\mathrm{BB}$ model with $\mathrm{kT} \approx 0.06$ $\mathrm{keV}$. The spectral properties of the source resemble those of Galactic and MC SSSs or black hole binary candidates.

The source is superposed on the emission-line object Ho 12. Existing data on this object argue that it may be either an H II region or a SNR. The variability of the x-ray source indicates that the x-rays are not due to direct emission from an SNR. We have identified a list of potential optical counterparts from the photometry of Gallart et al. (1996a), and are seeking to obtain follow-up spectroscopy of these objects. However, comparison with optical data for similar objects in the Galaxy and the MCs indicates that the source may be too faint to appear in existing optical data. Optical and UV imaging from HST is the best currently available means of searching for a counterpart. 
We are happy to thank Carme Gallart for access to her photometry and $I$-band image of NGC 6822, and Paul Hodge for allowing us to use his $\mathrm{H} \alpha$ data. Jack Hughes gave us advice on the x-ray properties of SNR, and Dan Harris on x-ray astrometry. Martin Elvis suggested that we include the $\chi^{2}$ confidence plots. Harley Thronson was the PI on the PSPC observing proposal for NGC 6822. We thank the referee, Niel Brandt, for a number of very useful suggestions. This research has made use of the NASA/IPAC Extragalactic Database (NED) which is operated by the Jet Propulsion Laboratory, Caltech, under contract with the National Aeronautics and Space Administration. This research was partially supported by the National Aeronautics and Space Administration under ROSAT Grant No. NAG 5-1973.

\section{REFERENCES}

Alcock, C., Allsman, R.A., Alves, D., Axelrod, T.S., Bennett, D.P., Charles, P.A., Cook, K.H., Freeman, K.C., Griest, K., Guern, J., Lehner, M.J., Livio, M., Marshall, S.L., Peterson, B.A., Pratt, M.R., Quinn, P.J., Rodgers, A.W., Southwell, K.A., Stubbs, C.W., \& Sutherland, W. 1997, MNRAS, 286, 483.

Arnaud, K.A. 1996 in "Astronomical Data Analysis Software and Systems V," ASP Conference Series v. 101, eds. G. Jacoby, \& J. Barnes (ASP: San Francisco) p. 17.

Beuermann, K., Reinsch, K., Barwig, H., Burwitz, V., de Martino, D., Mantel, K.-H., Pakull, M.W., Robinson, E.L., Schwope, A.D., Thomas, H.-C., Trümper, J., van Teeseling, A., \& Zhang, E. 1995, A\&A, 294, L1.

Bradt, H.D.V., Ohashi, T., \& Pounds, K.A. 1992, ARA\&A, 30, 391.

Collier, J., \& Hodge, P.W. 1994, ApJS, 92, 119; AAS CD-ROM Vol. 2.

Collier, J., Hodge, P.W., \& Kennicutt, R.C. 1995, PASP, 107, 361.

Cowley, A.P. 1992, ARA\&A, 30, 287.

Cowley, A.P., Schmidtke, P.C., Hutchings, J.B., Crampton, D., \& McGrath, T.K. 1993, ApJ, 418, L63.

Cowley, A.P., Schmidtke, P.C., McGrath, T.K., Ponder, A.L., Fertig, M.R., Hutchings, J.B., \& Crampton, D. 1997, PASP, 109, 21.

Crampton, D., Hutchings, J.B., Cowley, A.P., Schmidtke, P.C., McGrath, T.K., O’Donoghue, D., \& Harrop-Allin, M.K. 1996, ApJ, 456, 320.

de Vaucouleurs, G., de Vaucouleurs A., Corwin, H.G., Jr., Buta, R.J., Paturel, G., \& Fouqué, P. 1991, Third Reference Catalogue of Bright Galaxies (Springer-Verlag: New York). 
D’Odorico, S., Dopita, M.A., \& Benvenuti, P. 1980, A\&AS, 40, 67.

Eskridge, P.B. 1995, PASP, 107, 561.

Eskridge, P.B., White, R.E., III, \& Davis, D.S. 1996, ApJ, 463, L59.

Fabbiano, G., Kim, D.-W., \& Trinchieri, G. 1992, ApJS, 80, 531.

Fabbiano, G., Trinchieri, G., \& van Speybroeck, L. 1987, ApJ, 316, 127.

Freedman, W.L. 1985, ApJ, 299, 74.

Freedman, W.L. 1988, AJ, 96, 4.

Gallagher, J.S., III, Hunter, D.A., Gillett, F.C., \& Rice, W.L. 1991, ApJ, 371, 142.

Gallart, C., Aparicio, A., \& Vílchez, J.M. 1996a AJ, 112, 1928.

Gallart, C., Aparicio, A., Bertelli, G., \& Chiosi, C. 1996b AJ, 112, 1950.

Giacconi, R., Branduardi, G., Briel, U., Epstein, A., Fabricant, D., Feigelson, E., Forman, W., Gorenstein, P., Grindlay, J., Gursky, H., Harnden, F.R., Jr., Henry, J.P., Jones, C., Kellogg, E., Koch, D., Murray, S., Schreier, E., Seward, F., Tannanbaum, H., Topka, K., van Speybroeck, L, Holt, S.S., Becker, R.H., Boldt, E.A., Serlemitsos, P.J., Clark, G., Canizares, C., Markert, T., Novick, R., Helfand, D., \& Long, K. 1979, ApJ, 230, 540.

Gizis, J.E., Mould, J.R., \& Djorgovski, S. 1993, PASP, 105, 871.

Gottesman, S.T., \& Weliachew, L. 1977, A\&A, 61, 523.

Grevesse, N., \& Anders, E. 1989 in "Cosmic Abundances of Matter," AIP Conference Proceedings v. 183, (AIP Press: New York) p. 1.

Griffith, M.R., Wright, A.E., Burke, B.F., \& Ekers, R.D. 1994, ApJS, 90, 179.

Harris, D.E., Perley, R., \& Carilli, C. 1994 in "Astronomical Data Analysis Software and Systems III," ASP Conference Series v. 61, eds. D.R. Crabtree, R.J. Hanisch \& J. Barnes (ASP: San Francisco) p. 375.

Hodge, P.W. 1969, ApJS, 18, 73.

Hodge, P.W. 1977, ApJS, 33, 69.

Hodge, P.W., Kennicutt, R.C., \& Lee, M.G. 1988, PASP, 100, 917.

Hodge, P.W., Smith, T., Eskridge, P.B., MacGillivray, H., \& Beard, S. 1991, ApJ, 379, 621.

Hubble, E.P. 1925, ApJ, 62, 409.

Israel, F.P., Tacconi, L.J., \& Baas, F. 1995, A\&A, 295, 599.

Kahabka, P., Pietsch, W., \& Hasinger, G. 1994, A\&A, 288, 538. 
Kahabka, P. 1995, A\&A, 304, 227.

Klein, U., Gräve, R., \& Wielebinski, R. 1983, A\&A, 117, 332.

Long, K.S., Charles, P.A., Blair, W.P., \& Gordon, S.M. 1996, ApJ, 466, 750.

Long, K.S., Helfand, D.J., \& Grabelsky, D.A. 1981, ApJ, 248, 925.

Markert, T.H., \& Donahue, M.E. 1985, ApJ, 297, 564.

Massey, P., Armandroff, T.E., Pyke, R., Patel, K., \& Wilson, C.D. 1995, AJ, 110, 2715.

Mathewson, D.S., Ford, V.L., Dopita, M.A., Tuohy, I.R., Long, K.S., \& Helfand, D.J. 1983, ApJS, 51, 345.

Mathewson, D.S., Ford, V.L., Tuohy, I.R., Mills, B.Y., Turtle, A.J., \& Helfand, D.J. 1985, ApJS, 58, 197.

McAlary, C., Madore, B., McGonegal, R., McLaren, R., \& Welch, D. 1983, ApJ, 273, 539.

McClintock, J.E., Horne, K., \& Remillard, R.A. 1995, ApJ, 442, 358.

Ohta, K., Tomita, A., Saito, M., Sasaki, M., \& Nakai, N. 1993, PASJ, 45, L21.

Perrine, F. 1922, MNRAS, 82, 489.

Primini, F.A., Forman, W., \& Jones, C. 1993, ApJ, 410, 615.

Seward, F.D., Schmidt, B., \& Slane, P. 1995, ApJ, 453, 284.

Schmidtke, P.C., Cowley, A.P., Frattare, L.M., McGrath, T.K., Hutchings, J.B., \& Crampton, D. 1994, PASP, 106, 843.

Skillman, E. 1987, in "Star Formation in Galaxies," ed. C.L. Persson, NASA CP-2466, p. 263.

Smith, H.E. 1975, ApJ, 199, 591.

Snowden, S.L. 1996 BAAS, 28, 900.

Stark, A.A., Gammie, C.F., Wilson, R.W., Bally, J., Linke, R.A., Heiles, C., \& Hurwitz, M. 1992, ApJS, 79, 77.

Supper, R., Hasinger, G., Pietsch, W., Trümper, J., Jain, A., Magnier, E.A., Lewin, W.H.G., \& van Paradijs, J. 1997, A\&A, 317, 328.

Treves, A., Belloni, T., Corbet, R.H.D., Ebisawa, K., Falomo, R., Makino, F., Makashima, K., Maraschi, L., Miyamoto, S., \& Tanzi, E.G. 1990, ApJ, 364, 266.

Trinchieri, G., Fabbiano, G., \& Peres, G. 1988, ApJ, 325, 531.

Verbunt, F. 1993, ARA\&A, 31, 93.

Wang, Q., Hamilton, T., Helfand, D.J., \& Wu, X. 1991, ApJ, 374, 475. 
Wang, Q., \& Wu, X. 1992, ApJS, 78, 391.

White, N.E., Nagase, F., \& Parmar, A.N. 1995, in "X-Ray Binaries," ed. W.H.G. Lewin, J. van Paradijs, \& E.P.J. van den Heuvel (Cambridge U Press: Cambridge), p. 1.

Wilson, C.D. 1992a, AJ, 104, 1374.

Wilson, C.D. 1992b, ApJ, 391, 144.

Wilson, C.D. 1994, ApJ, 434, L11.

Wilson, C.D. 1995, AJ, 109, 449. 
Table 1 - PSPC Model Fitting Results

\begin{tabular}{lcccrcccc}
\hline \hline Model & $\begin{array}{c}\mathrm{kT} \\
\mathrm{keV}\end{array}$ & $90 \%$ conf. & $\begin{array}{c}N_{H} \\
10^{21} \mathrm{~cm}^{-2}\end{array}$ & $90 \%$ conf. & $\begin{array}{c}f_{X}{ }^{1} \\
0.2-2 \mathrm{keV}\end{array}$ & $\begin{array}{c}f_{X}{ }^{1} \\
0.2-4 \mathrm{keV}\end{array}$ & $\begin{array}{c}\mathrm{HRI}_{\text {Ein }}^{2} \\
10^{-3} \mathrm{~s}^{-1}\end{array}$ & $\chi^{2} / \nu$ \\
\hline R-S $_{h o t}$ & 0.56 & $0.090-0.79$ & 0.39 & $0.1-19.7$ & 8.25 & 8.38 & 1.30 & $16.8 / 17=0.99$ \\
R-S $_{\text {cool }}$ & 0.044 & $0.042-0.79$ & 22.3 & $18.9-27.6$ & 7.05 & 8.23 & 1.49 & $17.8 / 17=1.05$ \\
Blackbody & 0.064 & $0.029-0.14$ & 12.7 & $2.1-32.8$ & 7.28 & 7.84 & 1.47 & $17.4 / 17=1.03$ \\
\hline
\end{tabular}

1: Fluxes are given in units of $10^{-14} \mathrm{erg} \mathrm{cm}^{-2} \mathrm{~s}^{-1}$.

2: Predicted count-rate for the Einstein HRI. The observed Einstein HRI count-rate is $4.21 \times 10^{-3} \mathrm{sec}^{-1}$ (Fabbiano et al. 1992).

Abundances for all fits were fixed at 0.25 Solar.

Table 2 - X-Ray and Optical Emission-Line Source Positions

\begin{tabular}{lcc}
\hline \hline & $\begin{array}{c}\text { RA(J2000.0) } \\
\text { h m s }\end{array}$ & $\begin{array}{c}\text { Dec(J2000.0) } \\
\text { ' ' } "^{\prime \prime}\end{array}$ \\
\hline Ho 12 & 194456.9 & -144827 \\
X-Ray & 194456.7 & -144831 \\
\hline
\end{tabular}


Table 3 - Data on Potential Optical Counterparts

\begin{tabular}{lcccccccc}
\hline \hline ID & RA(J2000.0) Dec(J2000.0) offset & $B$ & $(U-B)$ & $(B-V)$ & $(V-R)$ & $(V-I)$ \\
\multicolumn{1}{c}{ h m s } & o' $^{\prime}$ & \multicolumn{1}{c}{ " } & & & & \\
\hline 17594 & 194457.2 & -144829 & 7.1 & $20.57 \pm 0.03$ & $0.66 \pm 0.09$ & & & \\
17633 & 194457.1 & -144825 & 8.4 & $21.61 \pm 0.07^{a}$ & & & & $1.21 \pm 0.11$ \\
$17683^{b}$ & 194457.1 & -144837 & 8.2 & $22.19 \pm 0.07$ & $-0.26 \pm 0.14$ & & & \\
$17801^{c}$ & 194457.0 & -144833 & 4.3 & $21.68 \pm 0.09$ & $-0.82 \pm 0.12$ & & & \\
17896 & 194456.9 & -144831 & 2.3 & $21.44 \pm 0.06$ & & & & \\
17913 & 194456.9 & -144822 & 9.5 & $22.52 \pm 0.11$ & $-0.53 \pm 0.16$ & & & \\
17996 & 194456.8 & -144829 & 2.0 & $21.60 \pm 0.07$ & & & & \\
$18013^{d}$ & 194456.7 & -144826 & 5.1 & $21.58 \pm 0.13$ & & & & \\
$18040^{e}$ & 194456.7 & -144841 & 9.5 & $21.09 \pm 0.05$ & $-0.23 \pm 0.08$ & & & \\
18075 & 194456.7 & -144824 & 7.5 & $22.59 \pm 0.12$ & $-0.36 \pm 0.19$ & $0.15 \pm 0.15-0.17 \pm 0.17$ & $0.48 \pm 0.16$ \\
18194 & 194456.5 & -144839 & 8.0 & $21.57 \pm 0.07^{a}$ & & & & \\
18260 & 194456.5 & -144829 & 3.9 & $21.53 \pm 0.08$ & $-0.10 \pm 0.17$ & $0.11 \pm 0.11$ & & $0.38 \pm 0.11$ \\
18332 & 194456.4 & -144832 & 4.5 & $22.50 \pm 0.10$ & $-0.56 \pm 0.17$ & & & \\
18435 & 194456.3 & -144839 & 9.6 & $20.67 \pm 0.14^{f}$ & & & & \\
18467 & 194456.3 & -144828 & 6.8 & $20.16 \pm 0.03$ & $-0.77 \pm 0.04$ & & & \\
18491 & 194456.3 & -144824 & 9.4 & $20.71 \pm 0.02$ & $0.10 \pm 0.08$ & $0.47 \pm 0.04$ & 0.390 .05 & $0.96 \pm 0.08$ \\
18630 & 194456.1 & -144835 & 9.8 & $20.70 \pm 0.02$ & $1.19 \pm 0.13$ & $1.31 \pm 0.04$ & 0.890 .05 & $1.74 \pm 0.06$ \\
\hline
\end{tabular}

${ }^{a}$ Magnitude is $V$-band.

${ }^{b}(B-R)=0.77 \pm 0.09,(B-I)=1.36 \pm 0.12$

${ }^{c}(B-I)=1.73 \pm 0.11$

${ }^{d}(B-I)=1.40 \pm 0.17$

${ }^{e}(B-I)=1.76 \pm 0.07$

${ }^{f}$ Magnitude is $I$-band. 
Fig. 1. - Top panel: The PSPC spectrum of the x-ray source (points with error bars), along with the best-fit warm $(\mathrm{kT} \approx 0.56 \mathrm{keV}) \mathrm{RS}$ model (solid line), and the best-fit ( $\mathrm{kT} \approx 0.06 \mathrm{keV}$ ) BB model (dotted line). Bottom panel: $\chi^{2}$ residuals of the fits to the data.

Fig. 2. $-\chi^{2}$ confidence contours of $\mathrm{kT}$ and $N_{H}$ for a) the $0.56 \mathrm{keV}$ RS model and b) the $0.06 \mathrm{keV} \mathrm{BB}$ model. The best-fit values are shown as plus signs. The contours are at the $1 \sigma, 90 \%$, and $99 \%$ confidence levels.

Fig. 3.- An $I$-band image of the vicinity of the x-ray source. North is up, East to the left. The center of the field is at the x-ray position given in Table 2. A 10" radius circle is plotted, containing the stellar objects listed in Table 3. The contours are $\mathrm{H} \alpha$ emission from Collier \& Hodge (1994) for the optical emission line objects Ho 12 and HK 65. 


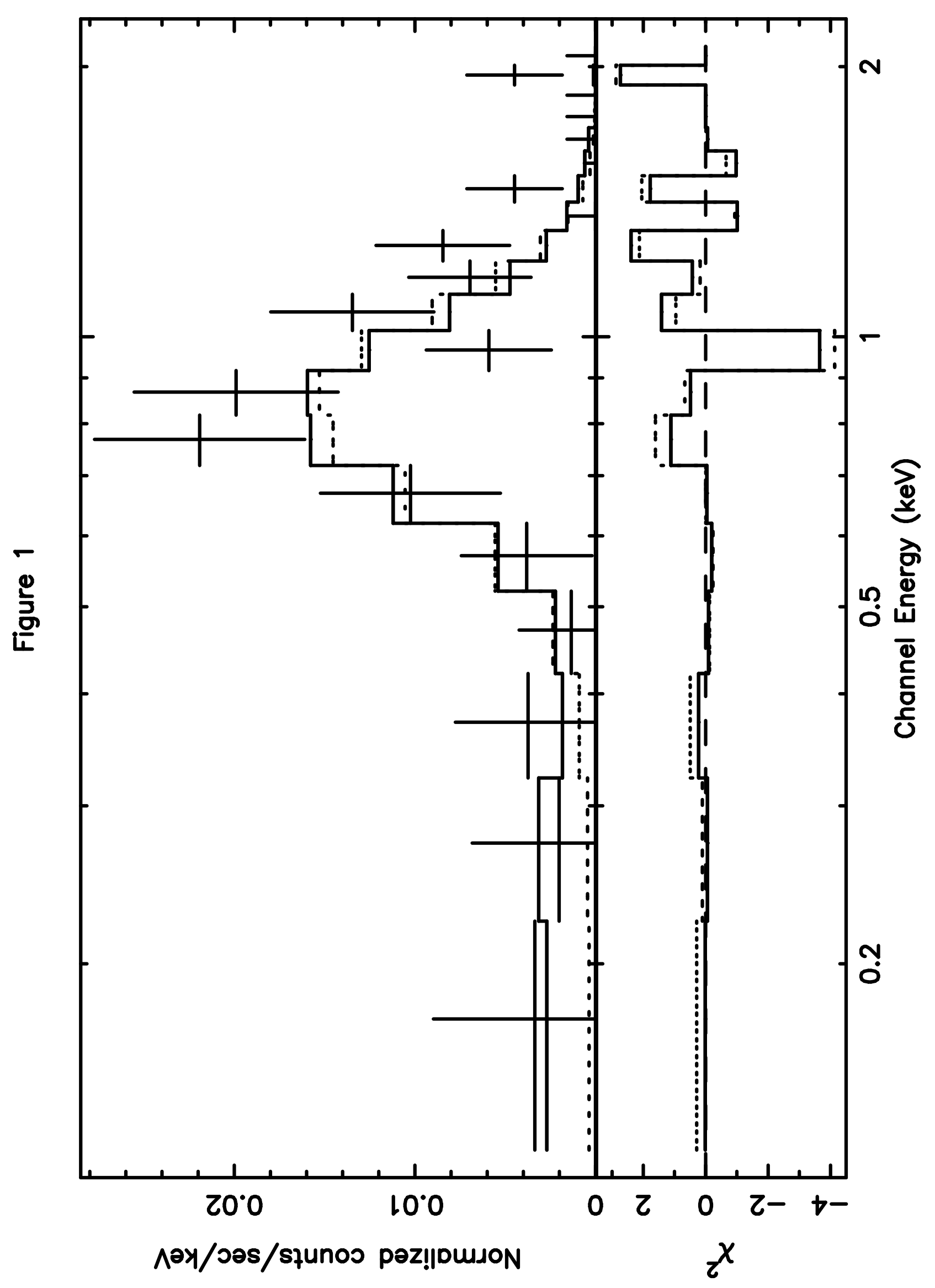




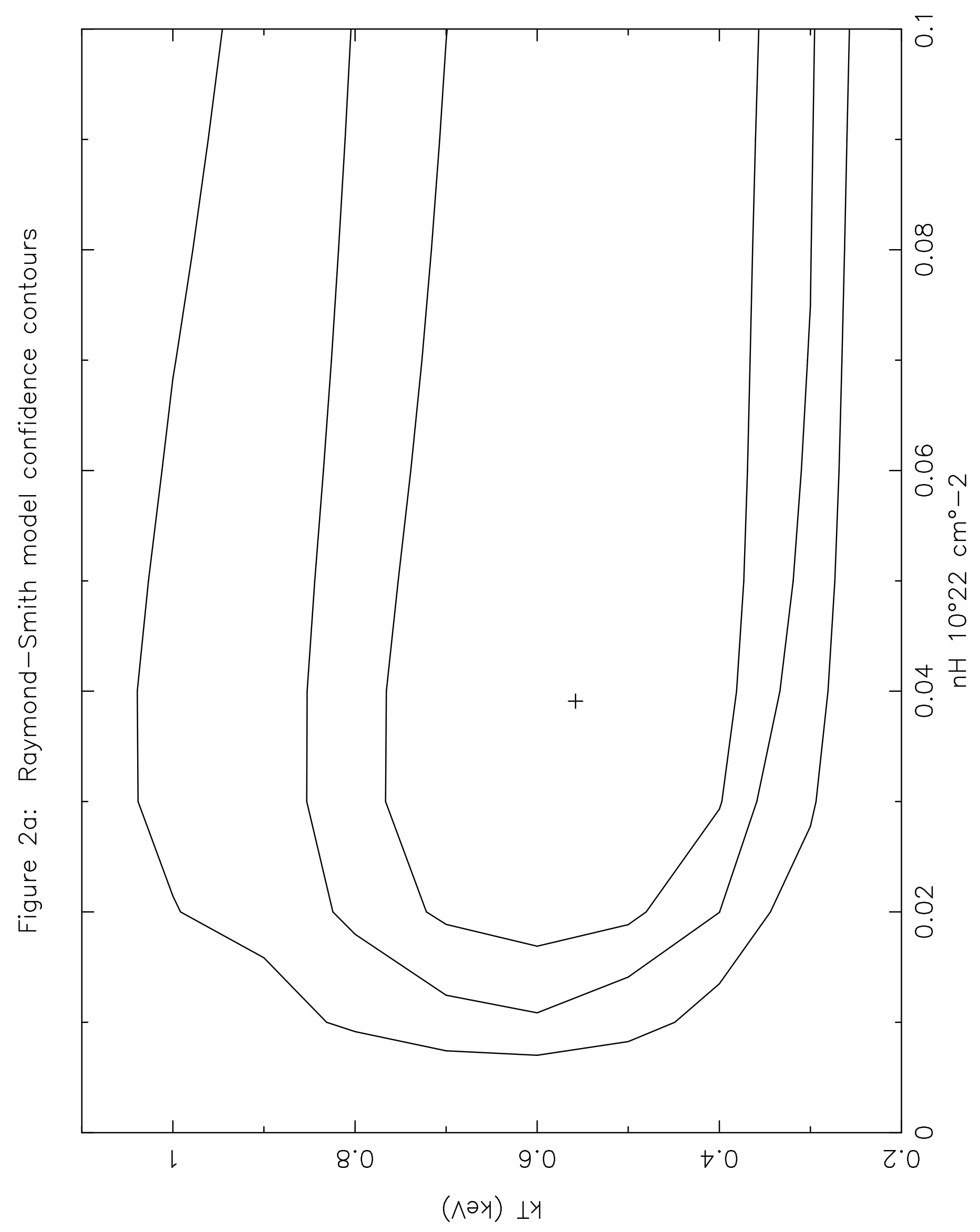




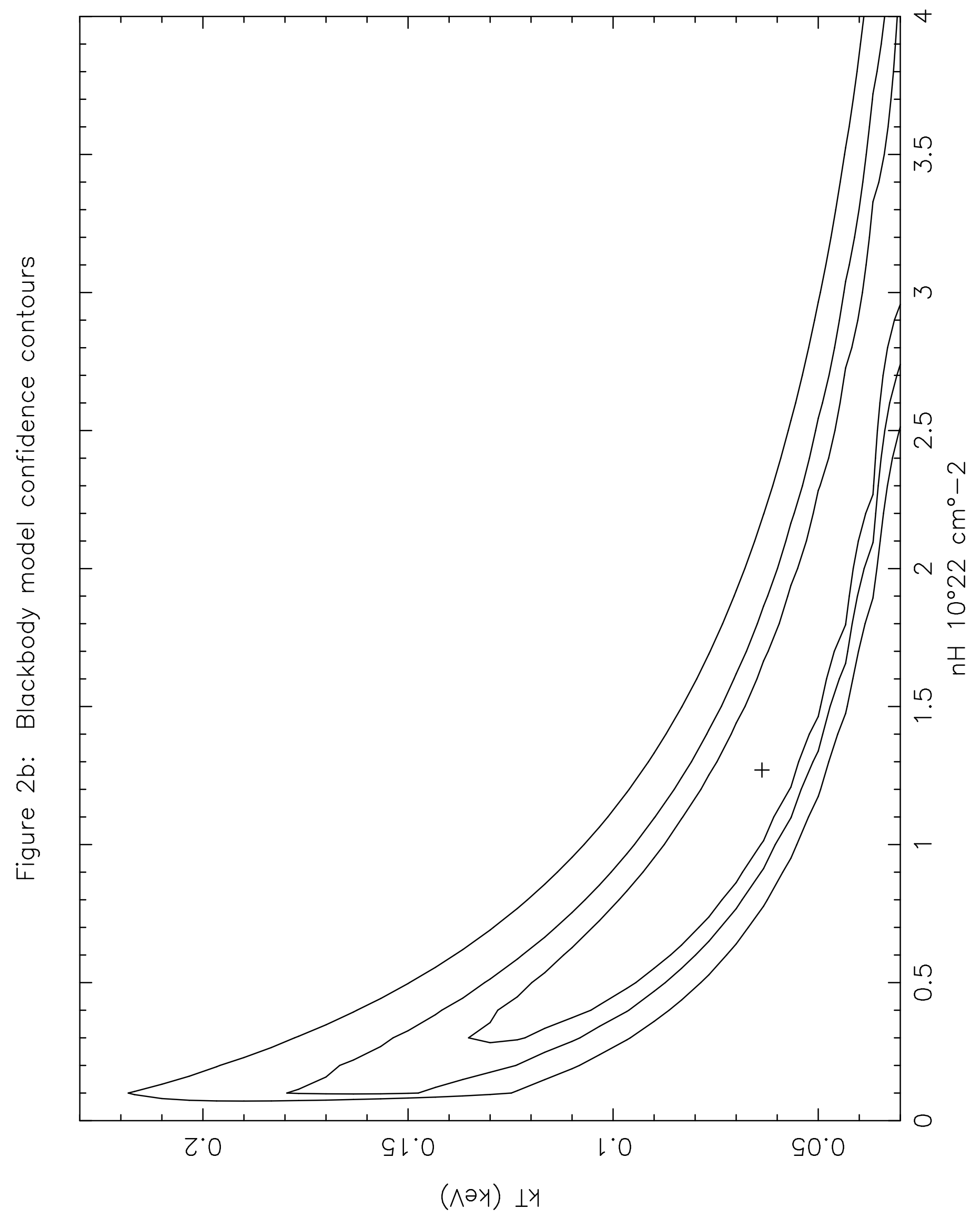


Figure 3

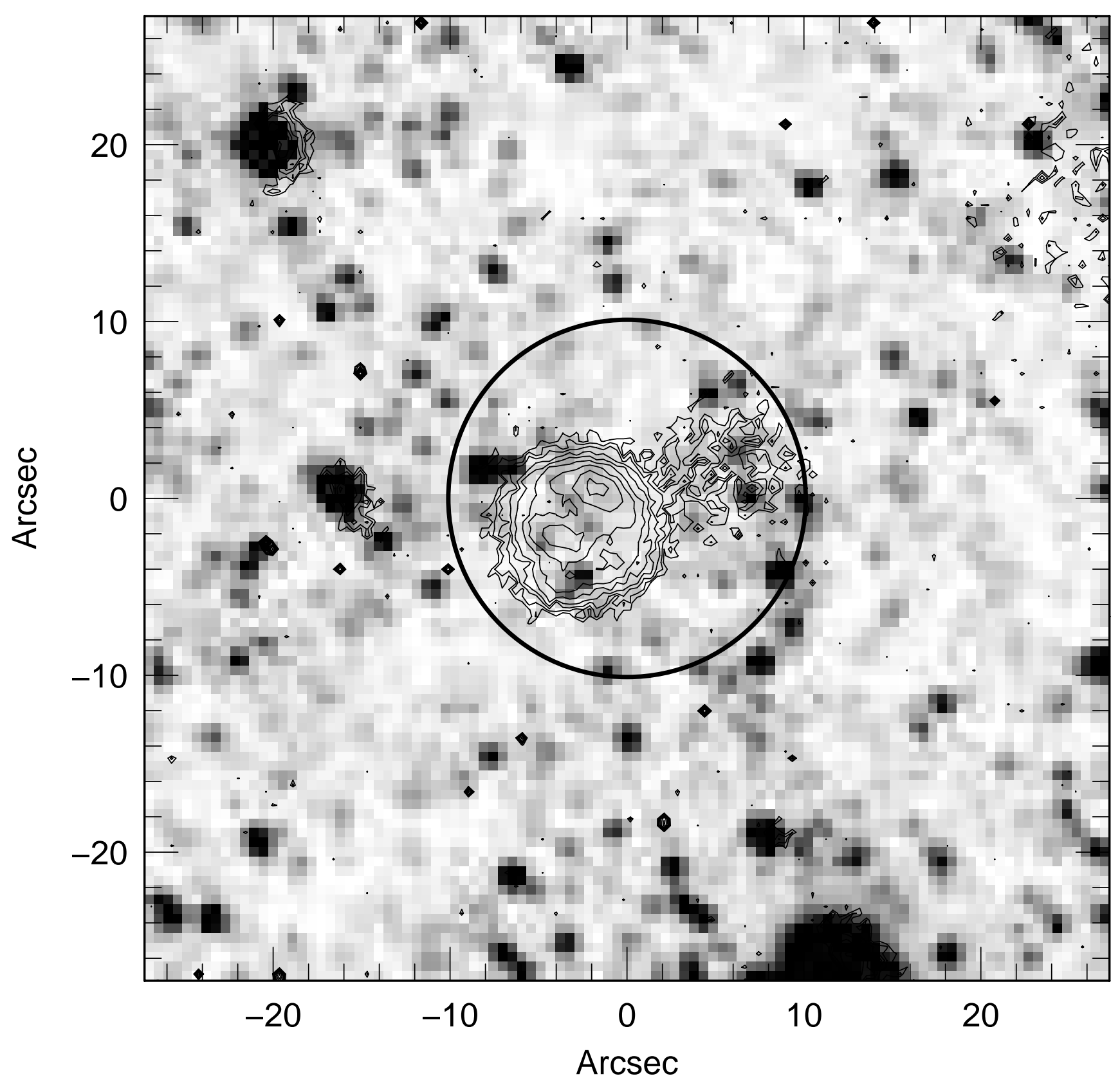

